\title{
Inherited Resistance to HIV-1 Conferred by an Inactivating Mutation in CC Chemokine Receptor 5: Studies in Populations with Contrasting Clinical Phenotypes, Defined Racial Background, and Quantified Risk
}

\author{
Peter A. Zimmerman, ${ }^{1}$ Alicia Buckler-White, ${ }^{5}$ Ghalib Alkhatib, ${ }^{2}$ \\ Todd Spalding, ${ }^{6}$ Joseph Kubofcik, ${ }^{1}$ Christophe Combadiere, ${ }^{3}$ \\ Drew Weissman, ${ }^{4}$ Oren Cohen, ${ }^{4}$ Andrea Rubbert, ${ }^{4}$ Gordon \\ Lam, ${ }^{4}$ Mauro Vaccarezza, ${ }^{4}$ Paul E. Kennedy, ${ }^{2}$ V. Kumaraswami, ${ }^{7}$ \\ Janis V. Giorgi, ${ }^{8}$ Roger Detels, ${ }^{9}$ Jay Hunter, ${ }^{6}$ Michael Chopek, ${ }^{6}$ \\ Edward A. Berger, ${ }^{2}$ Anthony S. Fauci, ${ }^{4}$ Thomas B. Nutman, ${ }^{1}$ \\ and Philip M. Murphy ${ }^{3}$ \\ Laboratories of ${ }^{1}$ Parasitic Diseases, ${ }^{2}$ Viral Diseases, ${ }^{3}$ Host Defenses, \\ and ${ }^{4}$ Immunoregulation, National Institute of Allergy and Infectious \\ Diseases, National Institutes of Health, Bethesda, Maryland, U.S.A. \\ ${ }^{5}$ Division of Molecular Virology and Immunology, Georgetown \\ University, Rockville, Maryland, U.S.A. ${ }^{6}$ National HLA \\ Histocompatibility Laboratory, American Red Cross, University of \\ Maryland Medical System, Baltimore, Maryland, U.S.A. \\ ${ }^{7}$ Tuberculosis Research Centre, Spur Tank Road, Chetput, Madras, \\ India ${ }^{8}$ Department of Medicine, UCLA School of Medicine, Los \\ Angeles, California, U.S.A. ${ }^{9}$ Department of Epidemiology, UCLA \\ School of Public Health, Los Angeles, California, U.S.A.
}

\begin{abstract}
Background: CC chemokine receptor 5 (CCR5) is a cell entry cofactor for macrophage-tropic isolates of human immunodeficiency virus-1 (HIV-1). Recently, an inactive CCR5 allele (designated here as CCR5-2) was identified that confers resistance to HIV-1 infection in homozygotes and slows the rate of progression to AIDS in heterozygotes. The reports conflict on the effect of heterozygous CCR5-2 on HIV-1 susceptibility, and race and risk

The nucleic acid sequence of CCR5-2 has been deposited in Genbank, \#U66285.

Address correspondence and reprint requests to: $\mathrm{Dr}$. Thomas B. Nutman, Laboratory of Parasitic Diseases, Building 4, Rm 126, National Institutes of Health, Bethesda, MD 20892-0425, U.S.A. or Dr. Philip M. Murphy, Laboratory of Host Defenses, Bldg. 10, Rm 11N-113, National Institutes of Health, Bethesda, MD 20892, U.S.A.
\end{abstract}

levels have not yet been fully analyzed. Here we report our independent identification of CCR5-2 and test its effects on HIV-1 pathogenesis in individuals with contrasting clinical outcomes, defined race, and quantified risk.

Materials and Methods: Mutant CCR5 alleles were sought by directed heteroduplex analysis of genomic DNA from random blood donors. Genotypic frequencies were then determined in (1) random blood donors from North America, Asia, and Africa; (2) HIV-1 + individuals; and (3) highly exposed-seronegative homosexuals with quantified risk.

Results: CCR5-2 was the only mutant allele found. It was common in Caucasians, less common in other North American racial groups, and not detected in West Africans or Tamil Indians. Homozygous CCR5-2 frequencies 
differed reciprocally in highly exposed-seronegative $(4.5 \%, n=111)$ and HIV-1-seropositive $(0 \%, n=614)$ Caucasians relative to Caucasian random blood donors $(0.8 \%, n=387)$. This difference was highly significant $(p<0.0001)$. By contrast, heterozygous CCR5-2 frequencies did not differ significantly in the same three groups $(21.6,22.6$, and $21.7 \%$, respectively). A $55 \%$ increase in the frequency of heterozygous CCR5-2 was observed in both of two cohorts of Caucasian homosexual male, long-term nonprogressors compared with other HIV-1 + Caucasian homosexuals $(p=0.006)$ and compared with Caucasian random blood donors. Moreover, Kaplan-Meier estimates indicated that CCR5-2 heterozygous seroconvertors had a $52.6 \%$ lower risk of developing AIDS than homozygous wild-type seroconvertors.

Conclusions: The data suggest that homozygous CCR5-2 is an HIV-1 resistance factor in Caucasians with complete penetrance, and that heterozygous CCR5-2 slows the rate of disease progression in infected Caucasian homosexuals. Since the majority $(\sim 96 \%)$ of highly exposed-seronegative individuals tested are not homozygous for CCR5-2, other resistance factors must exist. Since CCR5-2 homozygotes have no obvious clinical problems, CCR5 may be a good target for the development of novel antiretroviral therapy.

\section{INTRODUCTION}

Chemokine receptors are a group of 7-transmembrane domain proteins originally studied in the context of leukocyte chemotaxis (1). Recently, several chemokine receptors-CXCR4, CCR2B, CCR3, and CCR5 (designated according to a recent nomenclature convention)-have been shown to serve as HIV-1 cell-entry cofactors in vitro, acting in concert with $\mathrm{CD} 4$ and the gp120 viral envelope glycoprotein to mediate the first step in the human immunodeficiency virus-1 (HIV-1) life cycle, fusion of the viral envelope with the plasma membrane (2-12).

Different passaged strains of HIV-1 exhibit different chemokine receptor preferences, the result of sequence polymorphisms in gpl20. Socalled macrophage-tropic, or nonsyncytiuminducing strains, use CCR3 and CCR5 preferentially; these strains have properties similar to most primary isolates from infected individuals who have not yet progressed to AIDS $(13,14)$. So-called $\mathrm{T}$ cell line-tropic, or syncytium-inducing strains, use CXCR4 (previously referred to as fusin, HUMSTSR, and LESTR) and CCR3 preferentially; primary isolates from patients with advanced AIDS often have properties similar to these passaged strains. CCR2B, CCR5, and CXCR4 are all expressed on macrophages and $\mathrm{CD}^{+}{ }^{+} \mathrm{T}$ cells, whereas CCR3 is expressed on eosinophils (15-21). Primary isolates have been identified that can use all four of these receptors for cell entry $(4,5,8)$.

A role for CCR5 in susceptibility to HIV-1 infection has been supported recently by three groups who independently identified a mutant allele containing a 32 base pair deletion in the open reading frame (ORF) of the CCR5 gene that induces a frame shift, a premature stop codon, and loss of HIV-1 coreceptor activity in vitro $(10-12)$. We will refer to the mutant allele as CCR5-2 and the wild-type allele as CCR5-1. Sixteen and one percent of healthy European Caucasians were reported to be heterozygous and homozygous for CCR5-2, respectively; however, the mutant allele was not observed in Japanese or black Africans $(11,12)$. A large survey of the mutation in North Americans at risk for HIV-1 infection identified high prevalence in Caucasians and relatively low prevalence in AfricanAmericans; however, a systematic survey of the general population was not reported (12).

CCR5-2 was found in homozygous form by Liu et al. in two individuals (EU2 and EU3) who, as they had previously reported, remain uninfected despite high exposure to HIV-1 and whose peripheral blood mononuclear cells resist infection with macrophage-tropic HIV-1 in vitro $(10,22)$. In contrast, in 723 and 1,343 HIV-1+ individuals typed by Samson et al. and Dean et al., no individual homozygous for the mutant allele was identified, indicating that this genotype is a powerful restriction factor for HIV-1 transmission $(11,12)$. Samson et al. also reported a $35 \%$ decrease in heterozygotes among HIV-1 + individuals relative to seronegative controls, whereas Dean et al. reported no significant difference $(11,12)$. Dean et al. also reported that HIV-1+ CCR5-2 heterozygous individuals progress slightly less rapidly to AIDS compared with homozygous wild-type individuals in homosexual cohorts, but not in hemophilia cohorts (12). The risk levels of the cohorts of exposed- 
uninfected individuals have not been previously correlated with the CCR5 genotype.

We have independently identified the CCR5-2 allele and studied its effects on HIV-1 susceptibility and disease progression in individuals with contrasting clinical phenotypes, defined race, and quantified risk. In addition, we report a systematic and unbiased survey of CCR5 genotypes in North American racial groups.

\section{MATERIAL AND METHODS}

\section{Blood Sample Collection and Assignment of Clinical Phenotype}

All samples were from anonymous donors, collected under clinical protocols approved by the corresponding institutional review boards. Samples representing the general North American population were obtained from random blood donors from the American Red Cross and the Laboratory of Parasitic Diseases, National Institute of Allergy and Infectious Diseases $(n=$ 1,222 ). All donors were between the ages of 18 and 55 , and were classified by self-identified racial group. Samples from HIV-1-seropositive individuals were obtained from the Multicenter AIDS Cohort Study of homosexual men (MACS; $n=391,171$ of whom were seroconvertors) and from patients studied by the Laboratory of Immunoregulation/National Institute of Allergy and Infectious Diseases (LIR/NIAID; $n=325$ ). Patients from the LIR/NIAID group were predominantly homosexual men infected by sexual transmission. Long-term nonprogressors (LTNP), defined as asymptomatic HIV-1+ individuals who have not had an AIDS-defining illness, have not been treated with anti-retroviral therapy, and have a $\mathrm{CD} 4^{+} \mathrm{T}$ cell count $>500 / \mu \mathrm{L}$ that remained stable for at least 7 years (23), were obtained from both MACS $(n=56)$ and LIR/ NIAID $(n=32)$ patient groups. All LTNPs were homosexual men. MACS LTNPs were matched for age, race, and CD4 count at MACS visit \#3 to sets of moderately rapid progressors (decline of $>10 \mathrm{CD} 4$ cells/semester, the value for the 50th percentile of $\mathrm{CD} 4$ decline) and rapid progressors (decline of $>53 \mathrm{CD} 4$ cells/semester, the value for the 25th percentile) to form sets of MACS triplets, as previously described (23). The highly exposed-seronegative individuals were all HIV-1seronegative MACS participants with a history of receptive anal intercourse with multiple male sex partners $(n=125)$, but they were identified by two separate criteria. The first group ( $n=$ 103) was composed of individuals for whom samples were available that had risk scores in the upper tenth percentile of seronegative MACS participants. Risk score, defined by the number of anal receptive intercourse partners over 2.5 years prior to MACS visit \#2 (24), ranged from 43 to 501 for individuals in the top tenth percentile. The second group was composed of 28 men identified as seronegative MACS participants from whom HIV-1 was isolated at least once (25). Six of these latter individuals also had risk scores in the top tenth percentile, giving a total of 125 highly exposed-seronegative individuals studied $(24,25)$.

\section{DNA Extraction}

Human genomic DNA was purified from either $100 \mu$ l whole blood or $\sim 1$ million purified peripheral blood leukocytes by methods described previously (26).

\section{PCR Amplification}

PCR amplification of the CCR5 full-length open reading frame (ORF) was performed using the following two primers: CCR5-up: 5' - CCCAAGC TTACTCTCCCCGGGTGGAACAAGATG - $3^{\prime}$ and CCR5 down: 5' - GGTCTAGAGTCACCAGCCCA CTTGAGTCCGTG - $3^{\prime}$. These same primers were utilized to amplify a CCR5-like sequence from chimpanzee DNA to be used as an outgroup heteroduplex probe, a strategy used previously to reveal human genetic polymorphisms $(26,28)$. Amplification reactions were performed in a solution $(100 \mu \mathrm{l})$ containing $30 \mathrm{pmoles}$ each of the appropriate primers and a PCR buffer $(50 \mathrm{mM}$ $\mathrm{KCl}, 10 \mathrm{mM}$ Tris-HCL [pH 8.4]; $1.5 \mathrm{mM} \mathrm{MgCl}_{2}$; $175 \mathrm{mM}$ dATP, dGTP, dCTP, and dTTP, $10 \%$ glycerol); and 3 units Taq polymerase (PerkinElmer, Norwalk, CT). The conditions for amplification were $95^{\circ} \mathrm{C}$ for $1 \mathrm{~min}$ (denaturing), $65^{\circ} \mathrm{C}$ for 1 min (annealing), and $72^{\circ} \mathrm{C}$ for $1 \mathrm{~min}$ (extension) for 35 cycles.

\section{Directed Heteroduplex Analysis (DHDA)}

DHDA was performed as described previously (28). Briefly, $5 \mu \mathrm{l}$ of the unlabeled amplicon from human genomic DNA were mixed directly with $5 \mu$ l of the biotin-labeled CCR5 probe amplified from chimpanzee genomic DNA using a 5'-biotinylated CCR5-up primer (Operon Technologies, Alameda, CA) and an unbiotinylated 
CCR5-down primer. Following a 2-min incubation at $95^{\circ} \mathrm{C}$ to denature the $\mathrm{PCR}$ products, the reactions were cooled to $4^{\circ} \mathrm{C}$ (ramp time $=45$ $\mathrm{sec})$. Heteroduplex reactions $(7 \mu \mathrm{l})$ were mixed with sample dye buffer $(0.25 \%$ bromophenol blue; $0.25 \%$ xylene cyanol; $30 \%$ glycerol) and samples of $3.5 \mu \mathrm{l}$ were loaded onto a $6 \% \mathrm{Ge}$ nepage + (Amresco, Solon, $\mathrm{OH}$ ) polyacrylamide gel (2.7 M urea). Electrophoresis was performed on $50 \mathrm{~cm}$ sequencing gels at $40 \mathrm{mAmps}$ for 2.5-3 $\mathrm{hr}$ in $1 \times \mathrm{TBE}$. The temperature of the gel was maintained between $40^{\circ}$ and $45^{\circ} \mathrm{C}$. Detection of heteroduplex products formed with the biotinylated probe was performed following "contactblot" transfer to positively charged Biodyne-B nylon membranes (ICN Biochemicals, Cleveland, $\mathrm{OH}$ ) using the Phototope ${ }^{\mathrm{TM}}$ detection system (New England Biolabs, Beverly, MA) as described previously (26).

\section{Cloning of PCR Products}

Following amplification from human genomic DNA templates, PCR products showing novel heteroduplex mobility were cloned into pCR2.1 (Invitrogen, La Jolla, CA). Multiple clones from DNA derived from two North American Caucasians and two African-Americans were sequenced using both manual dideoxy-nucleotide chain termination sequencing and automated sequencing (ABI 373A, Applied Biosystems, Foster City, CA).

\section{Detection of PCR-amplified CCR5 Alleles}

Following PCR amplification of genomic DNA, 5 $\mu l$ were subjected directly to $B g l$ II digestion, and the products were visualized following electrophoresis on a $3 \%$ agarose gel $(5: 1$ GTG NuSieve:LE agarose) stained with ethidium bromide. Alternatively, following PCR amplification of human genomic DNA using a 5'-unbiotinylated CCR5-up and a biotinylated CCR5-down primer pair, ELISA-based detection of CCR5 amplicons was performed as described previously (29) modified only by the use of fluoresceinated (FL) sequence-specific oligonucleotide probes. The probe names are 5.1 ( $5^{\prime}$-FL-GTATCAATTCT GGAAGAATTTCCAG-FL-3'), which is specific for CCR5-1, and $5.2\left(5^{\prime}\right.$-FL-CATTTTCCATACAT TAAAGGTAGTC-FL-3'), which is specific for CCR5-2. A filter mat detection system was also developed using methods identical to those described previously (30), and probes 5.1 and 5.2 labeled with digoxigenin rather than fluorescein.
The results of these three different methods to detect CCR5-2 alleles were $>99 \%$ concordant.

\section{Cell Fusion Assay of HIV-1 Coreceptor Activity}

The CCR5-2 insert from clone 003.3 was excised by double digestion with Hind III and Xba I, blunt-ended, and subcloned into the Stu I site of pSC59, a vector containing the vaccinia virus early/late promoter (3). A pSC59 construct containing CCR5-1 has been previously described (3). Recombinant plasmid DNA was transfected into NIH-3T3 cells by lipofection (Boehringer Mannheim, Indianapolis, IN). The vaccinia promoter was activated by co-infection of the transfected cells with recombinant vaccinia viruses vCB-3 (encoding human CD4) and vTF7-3 (encoding T7 RNA polymerase). HeLa cells were co-infected with vCB-2l R (containing the lac $\mathrm{Z}$ gene encoding $\beta$-galactosidase, under control of the T7 promoter) and one of the following HIV-1 envelope glycoprotein (Env)-encoding vaccinia viruses: vCB-16 encoding the nonfusogenic UNC Env, vCB-4l encoding the T cell line-tropic LAV Env, or vCB-43 encoding the macrophage-tropic Ba-L Env (31). The dual-tropic 89.6 Env (cloned into pSC59 vector) was expressed on HeLa cells by lipofection and infection with vCB-21 R. The two types of cells were incubated separately overnight at $31{ }^{\circ} \mathrm{C}$ to allow synthesis of vaccinia virus-encoded proteins, then washed, resuspended at $10^{6}$ per $\mathrm{ml}$, and cocultured in duplicate for $2.5 \mathrm{hr}$ at $37^{\circ} \mathrm{C}$. Samples were then analyzed by a colorimetric lysate assay for $\beta$ galactosidase activity indicative of fusion of the T7 polymerase expressing NIH-3T3 cells with the lac Z-containing HeLa cells (3).

\section{Statistical Analysis}

The significance of differences in genotypic frequencies between different populations was tested by chi-square. The proportion of MACS seroconvertors remaining free of AIDS was computed using Kaplan-Meier estimates, and hypothesis tests of differences of survival curves were performed using Cox proportional-hazards models.

\section{RESULTS}

\section{Discovery of a Mutant CCR5 Allele}

After identifying the HIV-1 coreceptor activity for CCR5 in vitro (3), we next tested whether 


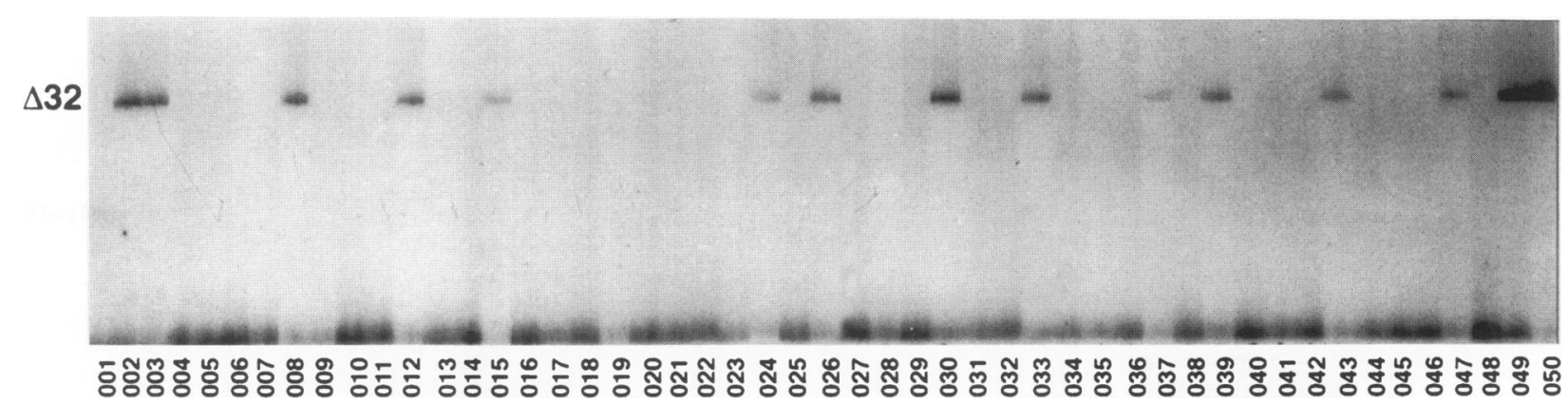

FIG. 1. Directed heteroduplex analysis of CCR5

Chemiluminescent contact blot of the directed heteroduplexed CCR5 amplicons from 50 random Caucasian blood donors. The numbers below each lane are the individual sample numbers. No other banding patterns were observed for 153 other samples tested (not shown).

CCR5 mutations might exist that could affect HIV-1 transmission and pathogenesis in vivo. The initial screen used the DHDA technique applied to the entire CCR5 ORF from 203 random blood donors. This strategy was possible since the ORF resides on a single exon $(20,21,32)$, allowing amplification en bloc by a single pair of PCR primers. DHDA can distinguish even single point mutations in a target sequence relative to a reference sequence based on differences in gel mobility of the resulting hybrid compared with the reference hybrid, a consequence of differences in secondary structure. The screen revealed only three band patterns (Fig. 1): (1) a single rapidly migrating band (e.g., individual \#001); (2) a single, slowly migrating band (e.g., individual \#050); and (3) two bands, one comigrating with the rapidly migrating band, the other comigrating with the slowly migrating band observed in the first two patterns (e.g., individual \#002). These results are consistent with either a second CCR5 allele containing major sequence differences in the ORF relative to the known allele, or another related gene or pseudogene.

Next, amplicons were cloned and sequenced from four unrelated individuals who had the two-band DHDA pattern; two were North American Caucasians and two were North American blacks. Two clones were sequenced from each of two of the individuals and one clone was sequenced from each of the others. Subclones from each of the four individuals were found that contained the identical 32 nucleotide deletion after nucleotide 552 of the reference sequence, where nucleotide 1 is the first nucleotide of the ORF. Outside of the deletion, one clone was identical in sequence to the reference sequence (clone 003.3), whereas the other five contained at most two base substitutions randomly located throughout the ORF, which is consistent with Taq polymerase-induced errors.

The 32 nucleotide deletion is identical to the mutation previously reported $(10-12)$. It results in a frame shift altering the deduced wild-type sequence beyond the predicted second extracellular loop of the receptor (Fig. 2A), a region shown to be critical for ligand binding by chemokine receptors (33). Moreover, it introduces a premature stop codon at nucleotide 678 of the reference sequence located in the region encoding the predicted third intracellular loop. Just upstream of the deletion resides a $\mathrm{Bgl}$ II site allowing for restriction fragment length polymorphism (RFLP) resolution of the wild-type and deleted sequences (Fig. 2B). Furthermore, we have developed allele-specific oligonucleotide probes that can be used in high throughput genotypic analysis using either 96-well plates (Fig. 2B) or filter mats (data not shown). Using these methods, we identified one individual in the group screened by heteroduplex analysis who was homozygous for the deletion, proving that the sequence containing the deletion resides at the functional locus and is not a pseudogene.

\section{Lack of HIV-1 Fusion-promoting Activity for CCR5-2}

We next examined the HIV-1 coreceptor activity of the mutant allele, CCR5-2, using a vacciniabased cell-cell fusion system (30). This assay models the first step in HIV-1 infection, envelope glycoprotein (Env)-dependent fusion of viral membrane with the membrane of target cells 


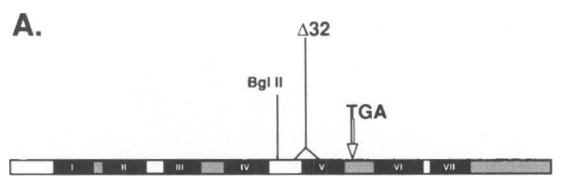

B.

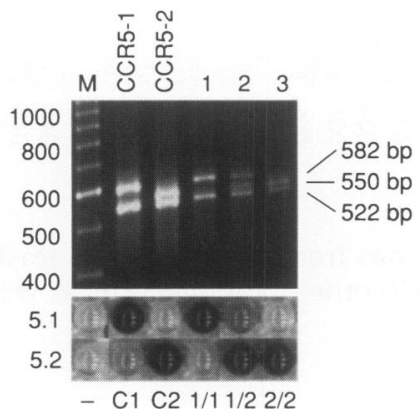

FIG. 2. Location and detection of CCR5 sequence polymorphisms

(A) Schema of the CCR5 ORF. Regions of the 1056 nucleotide ORF encoding transmembrane domains I-VII are shown in black, the proposed extracellular domains in white, and the intracellular domains are stippled in the linear scheme shown. The sites of the 32 nucleotide deletion $(\Delta 32)$ and a $B g l$ II restriction site are indicated. The double arrow indicates the position of the premature stop codon (TGA) found in CCR5-2. (B) Resolution of CCR5 alleles by restriction fragment length polymorphism analysis (top) and allele-specific PCR ELISA (bottom). For both analyses, the samples studied are indicated above the corresponding column coded as follows: M, 100 base pair interval markers; CCR5-1 and CCR5-2, plasmids containing CCR5-1 (wild type) and CCR5-2 (mutant) ORFs respectively; $1-3$, individual human genomic DNA samples. Genotypes are noted below the corresponding column of samples and coded as follows: $\mathrm{C} 1$ and $\mathrm{C} 2$ are the corresponding plasmid controls; 1/1, CCR5-1 homozygote; 1/2, CCR5-1/ CCR5-2 heterozygote; $2 / 2$, CCR5-2 homozygote. (Top) CCR5 amplicons were digested with Bgl II and analyzed by agarose gel electrophoresis. At the right are the lengths (in base pairs) of the indicated fragments. At the left are the lengths in base pairs of the marker standards. (Bottom) The ELISA-based PCR detection of the same samples. The allele specific oligonucleotide probes from CCR5-1 and CCR5-2 (5.1 and 5.2, respectively) are indicated to the left of the corresponding row of samples.

expressing CD4 and an appropriate coreceptor. Cells transfected with the CCR5-2 plasmid failed to fuse with the reporter targets, whereas cells transfected with a CCR5-1 plasmid exhibited strong fusion activity with partner cells expressing Envs from either the macrophage-tropic strain, Ba-L, or the dual-tropic isolate, 89.6 (Fig. 3). This result agrees with those of Liu et al.

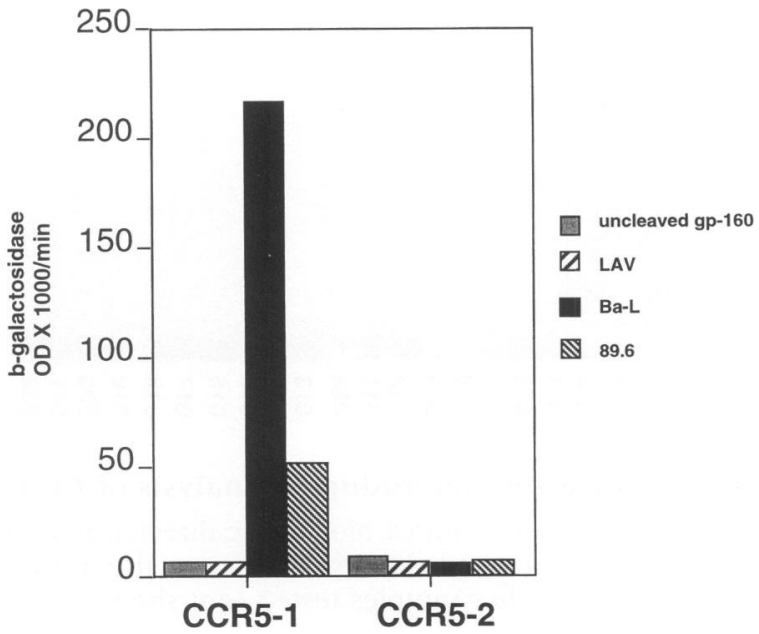

FIG. 3. Lack of HIV-1 coreceptor activity of CCR5-2

Plasmids containing DNA inserts indicated on the $\mathrm{x}$-axis were transfected into NIH $3 \mathrm{~T} 3$ cells expressing human CD4 and mixed with NIH 3T3 cells expressing the envelope glycoproteins from the HIV-1 strains coded to the right. Data are expressed as $\beta$-galactosidase activity.

and Samson et al. $(10,11)$.

\section{Distribution of CCR5 Alleles in Different Racial Groups}

The distribution of CCR5-2 in human populations was assessed by screening large numbers of anonymous blood donors of different racial backgrounds (Table 1). In North American Caucasians, the frequency of CCR5-2 heterozygotes was $21.7 \%$; it was much less common in AfricanAmericans (5.8\%), Hispanics of North American origin $(6.9 \%)$, and Asian-Americans $(0.6 \%)$. In Native North Americans the frequency of heterozygotes was $12.6 \%$. The deletion was not detected in West Africans or in South Indians. Seven CCR5-2 homozygotes were found in a total of 1,222 healthy adult blood donors tested, three North American Caucasians, three Native Americans, and one Hispanic individual of North American origin. Within each of the North American populations examined, with the possible exception of the Native Americans, the genotypic frequencies are observed to be in HardyWeinberg equilibrium.

\section{Effect of CCR5-2 on HIV-1 Susceptibility}

Because of the potential impact of CCR5-2 on HIV-1 pathogenesis, we also examined the fre- 
TABLE 1. Genotypic distribution of wild-type and mutant CCR5 alleles in different racial groups within general and HIV-1 exposed populations

\begin{tabular}{|c|c|c|c|c|c|c|c|c|}
\hline \multirow{2}{*}{$\begin{array}{l}\text { HIV-1 } \\
\text { Phenotype }\end{array}$} & \multirow[b]{2}{*}{ Region/Race ${ }^{a}$} & \multirow[b]{2}{*}{$\mathbf{n}$} & \multicolumn{6}{|c|}{ Genotypic Totals $^{b}$} \\
\hline & & & $1 / 1$ & $(\%)$ & $1 / 2$ & $(\%)$ & $2 / 2$ & (\%) \\
\hline \multirow{8}{*}{$\begin{array}{l}\text { Random blood } \\
\text { donors }\end{array}$} & NA/Caucasians & 387 & 300 & $(77.5)$ & 84 & $(21.7)$ & 3 & $(0.8)$ \\
\hline & NA/Blacks & 294 & 277 & $(94.2)$ & 17 & $(5.8)$ & 0 & $(0.0)$ \\
\hline & NA/Hispanics & 290 & 269 & $(92.8)$ & 20 & $(6.9)$ & 1 & $(0.3)$ \\
\hline & NA/Asians & 164 & 163 & $(99.4)$ & 1 & $(0.6)$ & 0 & $(0.0)$ \\
\hline & $\begin{array}{l}\text { NA/Native } \\
\text { Americans }\end{array}$ & 87 & 73 & $(83.9)$ & 11 & $(12.6)$ & 3 & (3.4) \\
\hline & WA/Blacks & 40 & 40 & $(100.0)$ & 0 & $(0.0)$ & 0 & $(0.0)$ \\
\hline & India Tamils & 46 & 46 & $(100.0)$ & 0 & $(0.0)$ & 0 & $(0.0)$ \\
\hline & Totals & 1308 & 1168 & & 133 & & 7 & \\
\hline \multirow[t]{4}{*}{ HIV-1+ } & NA/Caucasians & 614 & 475 & $(77.4)$ & 139 & $(22.6)$ & 0 & $(0.0)$ \\
\hline & NA/Blacks & 86 & 84 & (97.7) & 2 & $(2.3)$ & 0 & $(0.0)$ \\
\hline & NA/Hispanics & 45 & 42 & $(93.3)$ & 3 & $(6.7)$ & 0 & $(0.0)$ \\
\hline & Totals & 745 & 601 & & 144 & & 0 & \\
\hline \multirow{4}{*}{$\begin{array}{l}\text { HIV-1 exposed/ } \\
\text { uninfected }\end{array}$} & NA/Caucasians & 111 & 82 & $(73.9)$ & 24 & $(21.6)$ & 5 & $(4.5)$ \\
\hline & NA/Blacks & 2 & 1 & $(50.0)$ & 1 & $(50.0)$ & 0 & $(0.0)$ \\
\hline & NA/Hispanics & 12 & 11 & $(91.7)$ & 1 & $(8.3)$ & 0 & $(0.0)$ \\
\hline & Totals & 125 & 94 & & 26 & & 5 & \\
\hline
\end{tabular}

${ }^{a} \mathrm{NA}=$ North America; WA $=$ West Africa.

${ }^{b} 1 / 1=$ CCR5-1 (wild-type allele) homozygotes; $1 / 2=$ CCR5-1/CCR5-2 heterozygotes; $2 / 2=$ CCR5-2 (deleted allele) homozygotes.

quency of CCR5-2 in HIV-1+ individuals of different racial backgrounds. CCR5-2 heterozygotes represented $22.6 \%$ of 614 North American Caucasians, $2.3 \%$ of 86 African-Americans, and $6.7 \%$ of 45 Hispanics of North American origin tested who were HIV-1+. These values did not differ significantly from those found in each of the respective racial subgroups of blood donors from the general population. No CCR5-2 homozygotes were identified in the HIV-1 + group.

We next assessed the CCR 5 genotypic distribution in 125 highly exposed-seronegative MACS participants, at risk for HIV-1 from frequent receptive anal intercourse with multiple male partners. Quantitative risk scores were available for all of these individuals, based on the number of different anal receptive intercourse partners before MACS visit \#2 (24). The individuals were identified in two ways: 103 were identified as those individuals for whom samples were available that had risk scores in the top tenth percentile of all seronegative MACS participants. An additional 28 were tested because they had been previously reported to be transiently infected with HIV-1, suggesting inherent resistance $(25) ; 6$ of these individuals also had risk scores in the top tenth percentile. For reference, the distribution of risk for the entire seronegative MACS cohort followed until at least $1990(n=2430)$ is skewed markedly to the left with a median of 5 partners, 25th percentile of 16, and 90th percentile of 42 (Fig. 4A). The distribution of risk for MACS seropositive individuals ( $n=2744$ ) is superimposable with that for the seronegative MACS participants (not shown). The great majority of the highly exposeduninfected individuals were Caucasian $(n=111$; $89.3 \%$ ) and had the homozygous wild-type genotype $(73.9 \%)$. The percentage of CCR5-2 heterozygotes found $(21.6 \%)$ was similar to that found in the general Caucasian population of random blood donors $(21.7 \%)$ and in the 

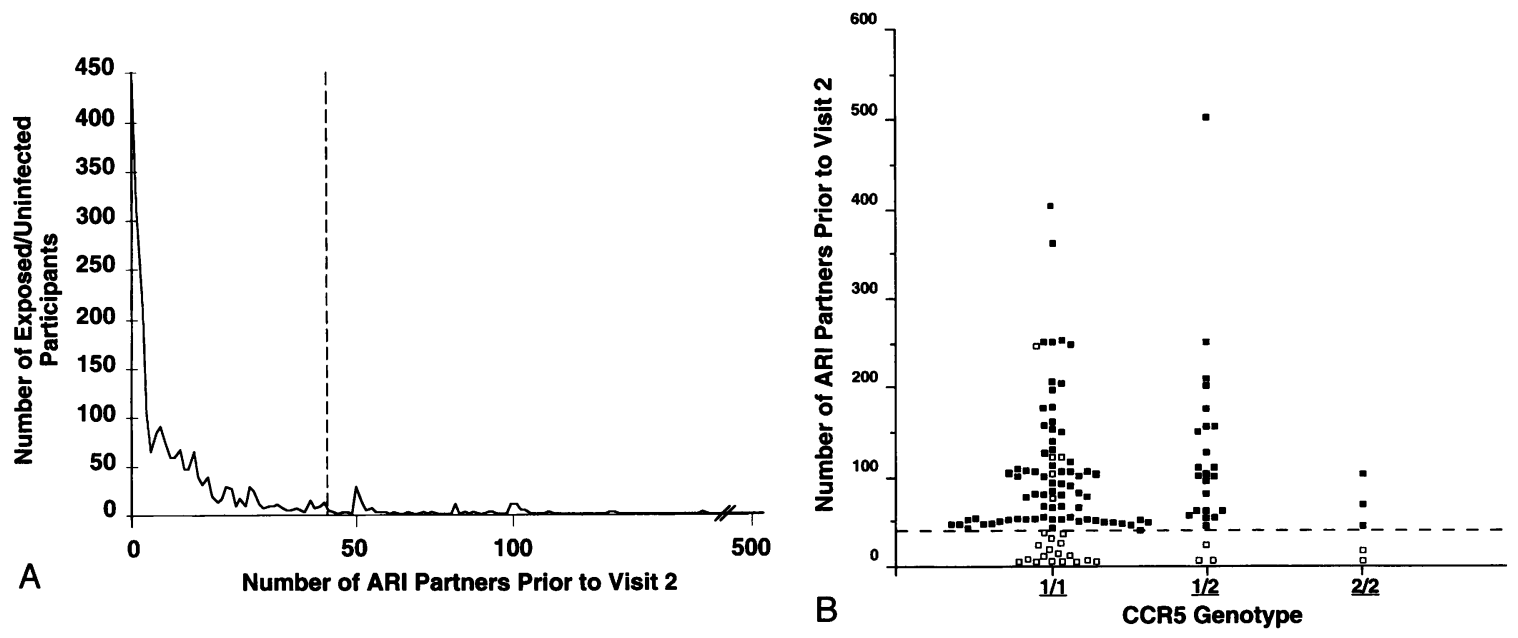

FIG. 4. Distribution of CCR5 genotypes by the degree of exposure to HIV-1 among highly exposedseronegative homosexuals

(A) Distribution of risk for all seronegative MACS participants ( $n=2430$ as of 10/96). Risk score is the number of anal receptive intercourse (ARI) partners over 2.5 years prior to MACS visit \#2 (24). The dotted vertical line demarcates the boundary between the top 10th and bottom 90th percentiles of risk. The distribution of risk for seropositive MACS participants did not differ significantly from that shown for seronegative MACS participants (not shown). (B) Distribution of CCR5 genotypes among highly exposed-seronegative MACS participants. CCR5 genotypes are coded as follows: 1/1, CCR5-1 homozygote; 1/2, CCR5-1/CCR5-2 heterozygote; 2/2, CCR5-2 homozygote. The dotted horizontal line demarcates the boundary between the top 10th and bottom 90 th percentiles. Each symbol corresponds to a single seronegative MACS participant with the corresponding genotype and risk score. Open symbols, individuals previously reported to be transiently infected with HIV-1 (25).

HIV-1 + Caucasians (22.6\%; Table 1). Five CCR5-2 homozygotes were detected in the highly exposed-seronegative group, a 6-fold increase over the frequency found for Caucasian random blood donors. The differences in genotypic frequency observed between this group and the HIV-1 + group were highly significant (chisquare, 2 degrees of freedom $=27.85, p<0.0001$ ), owing to reciprocal partitioning of CCR5-2 homozygotes relative to expected frequencies (Table 2). The risk scores for each of the highly exposed-uninfected individuals tested are shown in relation to their CCR5 genotype in Fig. 4B. Three of these had risk scores in the top tenth percentile. The two that had lower risk scores were both from the group of transiently HIV-1-infected individuals.

TABLE 2. Comparison of CCR5 genotypic distribution among general and HIV-1 exposed Caucasian populations

\begin{tabular}{lccc}
\hline HIV-1 Phenotypes & $\chi^{2}$ & d.f. & $a_{p \text {-value } \cong}$ \\
\hline EU vs. HIV-1+ & 27.85 & 2 & $<0.0001$ \\
EU vs. Normals & 7.62 & 2 & 0.022 \\
HIV-1 + vs. Normals & 4.85 & 2 & 0.088 \\
LTNP vs. HIV-1+ & 7.42 & 1 & 0.006 \\
LTNP(a) vs. LTNP(b) ${ }^{b}$ & 0.0015 & 1 & 0.969 \\
\hline
\end{tabular}

$\mathrm{EU}=$ HIV-1 exposed/uninfected individuals; Normals = random blood donors; LTNP = long-term nonprogressors; d.f. $=$ degrees of freedom.

${ }^{a} \cong p$-values are uncorrected. Application of Bonferroni correction factor would multiply all $p$-values by 5 accounting for the 5 comparisons performed.

${ }^{b}$ LTNP (a) was obtained from MACS $(n=53)$; LTNP(b) was obtained from LIR/NIAID $(n=32)$. 
TABLE 3. CCR5 genotypes in sets of matched $^{a}$ seropositive Caucasians exhibiting discordant rates of disease progression

\begin{tabular}{llrrr}
\hline \multicolumn{2}{l}{ Genotype Combinations within Matched Triplets } & & \\
\hline $\mathbf{R}^{b}$ & $\mathbf{M}$ & $\mathbf{L}$ & $\mathbf{n}^{c}$ & \% $^{d}$ \\
\hline $1 / 1$ & $1 / 1$ & $1 / 2$ & 8 & $(16.7)$ \\
$1 / 1$ & $1 / 2$ & $1 / 2$ & 4 & $(8.3)$ \\
$1 / 2$ & $1 / 2$ & 1 & $(2.1)$ \\
$1 / 2$ & $1 / 2$ & $1 / 2$ & 4 & $(8.3)$ \\
$1 / 1$ & $1 / 1$ & $1 / 1$ & 3 & $(6.3)$ \\
$1 / 1$ & $1 / 2$ & $1 / 1$ & 20 & $(41.7)$ \\
$1 / 2$ & $1 / 1$ & $1 / 1$ & 5 & $(10.4)$ \\
$1 / 2$ & $1 / 1$ & $1 / 1$ & 3 & $(6.3)$ \\
& $1 / 2$ & & 48 & $(100.0)$
\end{tabular}

\footnotetext{
${ }^{a}$ Seropositive MACS participants concordant for multiple clinical criteria but discordant for rate of progression to AIDS (22). ${ }^{b} \mathrm{R}=$ rapid; $\mathrm{M}=$ moderately rapid; $\mathrm{L}=$ long-term nonprogressor as defined by the rate of $\mathrm{CD} 4^{+}$leukocyte change (22). ${ }^{c} \cong n=$ number of triplets exhibiting CCR5 genotype combination.

${ }^{d} \%=$ percentage of triplets exhibiting CCR5 genotype combination .
}

\section{Effect of CCR5-2 on Disease Progression in HIV-1-infected Individuals}

We next determined the genotypic distribution in Caucasian HIV-1 + long-term nonprogressors (LTNPs). No LTNPs homozygous for CCR5-2 were found. An almost identical proportion of heterozygotes was observed for both of two separate groups of LTNPs, $34.0 \%$ for the MACS group and $34.4 \%$ for the LIR/NIAID group. This represents a $55 \%$ increase in the heterozygous genotype relative to both the rest of the Caucasian HIV-1+ individuals tested and the Caucasian random blood donors. The difference in genotype frequency observed between all Caucasian LTNPs $(n=85)$ and the rest of the HIV-1 + Caucasians $(n=529)$ was statistically significant (chi-square, 1 degree of freedom $=$ 7.42, $p=0.006$ ) (Table 2).

The MACS cohort of LTNPs has previously been matched with moderately rapid and rapid progressors by multiple epidemiologic criteria (see Materials and Methods) to form 56 sets of matched triplets, 48 of which had only Caucasian members (23). The distribution of the eight possible genotype combinations for the Caucasian triplets is shown in Table 3. Most members (106/ 144) were homozygous for the wild-type CCR5-1 allele. CCR5-2 heterozygous individuals were found in 28 of the 48 triplets. Their distri- bution in the three progression categories was: LTNPs, 17; moderately rapid progressors, 8; and rapid progressors, 13. Eleven triplets had two heterozygous members, and one triplet had three heterozygous members. Twelve triplets were biased in favor of a relationship of the heterozygous CCR5-2 genotype with slower progression (that is, triplets having only a nonprogressor member or both a moderate progressor and a nonprogressor member, but not a rapid progressor member, who are heterozygous for CCR5-2), and eight triplets were biased in favor of rapid progression (that is, triplets having only a rapid progressor member or both a moderate progressor and a rapid progressor member, but not a nonprogressor member, who are heterozygous for CCR 5-2). Among the triplets with heterozygous members, differences in the observed and expected frequencies of CCR5 genotypes did not reach statistical significance $(0.4>p>0.3)$.

The Kaplan-Meier life table curve of AIDSfree survival was shifted to the right for MACS seroconvertors having the CCR5-2 heterozygous genotype $(n=34)$ versus the homozygous CCR5-1 wild-type genotype ( $n=137$; Fig. 5). A Cox proportional hazards model of the difference between the two curves indicated that homozygous wild-type seroconvertors have a $52.6 \%$ 


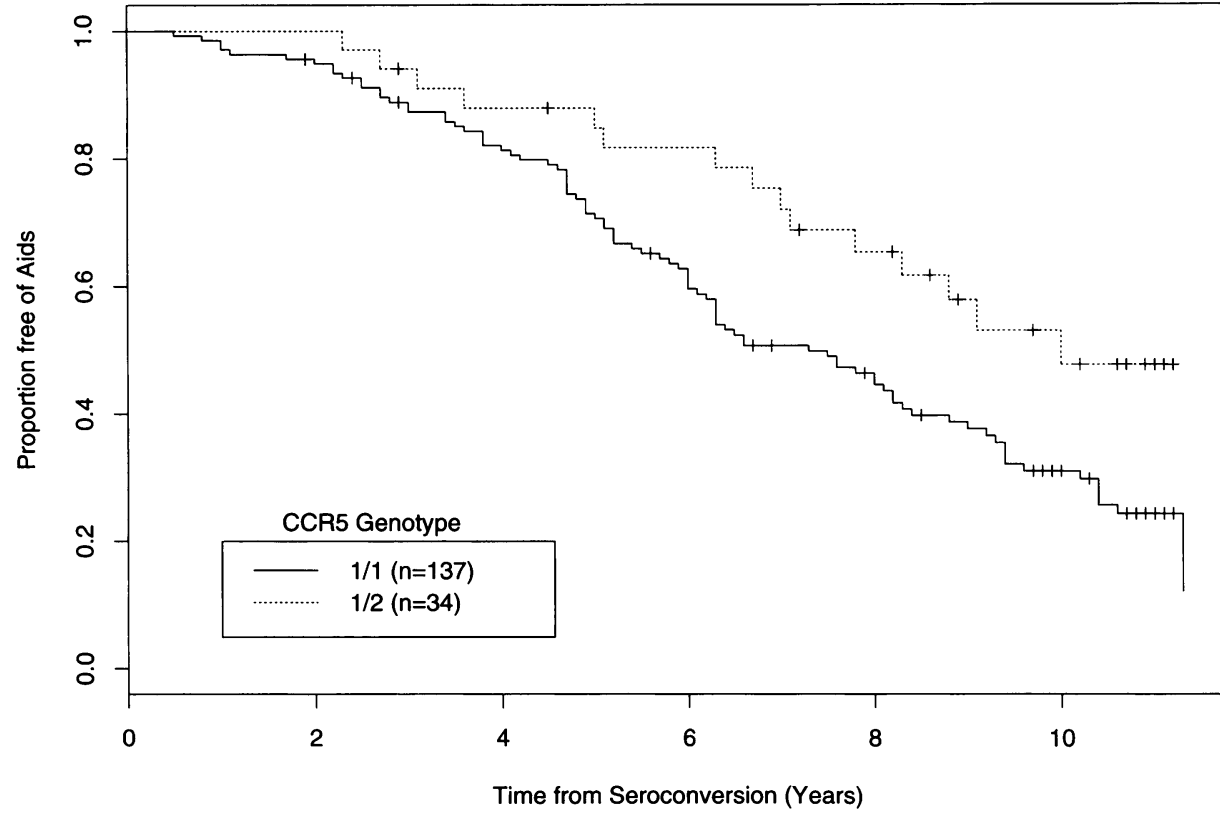

FIG. 5. Effect of CCR5 genotype on rate of progression to AIDS Kaplan-Meier life table curves are shown for all seroconvertors tested from the MACS cohort $(n=$ 171), segregated by CCR5 genotype. greater hazard or risk of developing AIDS than the heterozygous seroconvertors (relative hazard $=0.526 ; p=0.013,95 \%$ confidence interval, 0.304-0.909). When the data were analyzed separately for the individual centers contributing patients to the study, variability from center to center was found.

\section{DISCUSSION}

Based on DHDA, an unbiased method for detecting DNA sequence polymorphism (28), the present study confirms the existence of at least two alleles for the CCR5 gene. The CCR5-1 allele encodes a receptor that functions physiologically as a recognition site for the CC chemokines MIP$1 \alpha$, MIP- $1 \beta$, and RANTES $(20,21,32)$, and pathologically as a cell entry cofactor for macrophagetropic strains of HIV-1 (2-6). CCR5 appears to be the target for suppression of macrophage-tropic HIV- 1 replication by MIP- $1 \alpha$, MIP- $1 \beta$, and RANTES $(2-4,6,34)$. The CCR5-2 allele contains a 32 nucleotide deletion in the region encoding the second extracellular loop, resulting in frame shift, a premature stop codon, and loss of HIV-1 coreceptor activity. These results agree with those recently reported by Liu et al. and Samson et al. $(10,11)$.

Our data show that the CCR5-2 allele is very common in North American Caucasians, but absent in Tamil Indians and West African blacks.
The relatively low frequency of CCR5-2 in African-Americans and Hispanics from North America and its rarity in Asian-Americans may reflect genetic admixture of emigrant populations with Caucasians in whom the mutation most likely originated. Our results are consistent with and extend those of Samson et al. who did not detect the allele in Japanese or Africans, whereas 16\% and $1 \%$ of European Caucasians were CCR5-2 heterozygotes and homozygotes, respectively (11).

The discovery of healthy adult blood donors homozygous for CCR5-2 suggests that a functional CCR5 allele is not essential for development. Furthermore, the absence of a significant difference between observed and expected numbers of CCR 5-2 homozygotes in the populations tested suggests that normal CCR5 function is dispensable, perhaps the result of the extensive overlap of chemokine and leukocyte selectivity found within the family of CC chemokine receptors (1). This is consistent with the absence of obvious health problems in the two exposeduninfected CCR5-2 homozygotes previously reported (10). Additional studies will be needed to examine the role of CCR5-2 in diseases that occur after development and reproduction, particularly chronic inflammatory diseases such as atherosclerosis.

The factors that fixed CCR5-2 in the Caucasian population await elucidation; perhaps other microbial opportunists that could use wild-type 
CCR5-1 but not CCR5-2 for pathogenesis during the course of major ancestral epidemics were responsible. This concept has a parallel in the relationship between Plasmodium vivax, a causative agent of malaria, and expression on red cells of the Duffy antigen, another chemokine receptor subtype (35-37). Indeed, it is believed that, under a process of gene inactivation for Duffy caused by the selective pressure of malaria, the majority of individuals descended from $P$. vivax-endemic areas now lack Duffy expression on their red cells and are resistant to vivax malaria. Although CCR5 acts as a susceptibility factor to HIV-1 infection in vivo, the resultant selective pressure is too recent (given the relatively recent emergence of HIV-1) in evolutionary time to account for fixation of CCR5-2 in the general population.

Our genotype data showing a similar frequency of heterozygous CCR5-2 for Caucasian random blood donors and for both HIV-1 + and exposed-uninfected Caucasians strongly suggest that CCR5-2 heterozygotes are equally susceptible to HIV-1 infection as are wild-type homozygotes. This agrees with the report of Dean et al. (12) but differs from Samson et al. (11) who reported a $35 \%$ reduction in the frequency of CCR5-2 heterozygotes in seropositive Caucasians from Europe compared with presumed seronegative individuals from a similar geographic origin and having a European patronyme $(10.8 \%$ versus $16.2 \%)$. Our method compared large numbers of random blood donors versus HIV-1+ individuals drawn from multiple centers around the United States. Additional typing of different European regions will be necessary to refine the precise genotypic frequencies that have been measured so far and to resolve the differences in genotypic frequency observed in Europe versus North America.

The absence of CCR5-2 homozygotes among the 614 HIV-1 + Caucasians whom we tested is consistent with the reports of Samson et al. and Dean et al. who tested 723 and 1,343 HIV-l+ individuals from Europe and North America, respectively $(11,12)$. These data, together with Dean et al.'s report of 17 CCR5-2 homozygotes among 612 seronegatives tested, and our observation of a 6-fold increase in the frequency of CCR5-2 homozygotes in the group of highly exposed-seronegative individuals strongly suggest that the CCR5-2 homozygous genotype is an HIV-1 resistance factor with complete penetrance. The magnitude of HIV-1 resistance conferred by this genotype appears to be very high, as reflected in the high risk scores of three of the highly exposed-seronegative CCR5-2 homozygous individuals, placing them in the top tenth percentile of risk for all MACS participants (Fig. 4B). It is also of interest that the other two exposed-seronegative CCR5-2 homozygotes were from the 22 exposed-seronegative MACS donors who were genotyped because they had been reported to be transiently viremic with HIV-1, suggesting innate resistance (25).

Conversely, our data indicate that the great majority of highly exposed-seronegative individuals tested lack the homozygous CCR5-2 genotype, implying that other resistance factors must exist. In fact, most of the CCR5-2 heterozygous and homozygous wild-type individuals in this group had risk scores that exceeded that of the CCR5-2 homozygous individual with the highest risk score. A search for additional polymorphisms in the CCR5 gene affecting receptor structure or expression, as well as in the other known HIV-l coreceptors may provide additional insights into the role of genetic factors in $\mathrm{HIV}-\mathrm{l}$ resistance. In this regard, it is interesting to note that the site of the 32 nucleotide deletion in CCR5-2 overlaps with the site of a 12 nucleotide deletion in CCR2B relative to the sequence of CCR5-1 $(20,21,32)$ and is found in a region of great variation in sequence length for other members of the 7-transmembrane domain receptor superfamily, of which the chemokine receptors form one of many branches.

Our results showing a highly statistically significant $55 \%$ increase in frequency of individuals heterozygous for CCR5-2 in both of two cohorts of Caucasian homosexual LTNPs, compared with other HIV-1 + Caucasians and Caucasian random blood donors, suggest that this genotype can affect the rate of clinical progression in HIV-1 + gay men. It is important to point out, however, that the majority of LTNPs had the CCR5 wild-type genotype, and that many CCR5-2 heterozygotes are rapid progressors. In the context of the MACS triplets, who are matched for age, race, and initial CD4 count, a skewing of the CCR5-2 heterozygous genotype towards slower progression was observed. However, the difference did not reach statistical significance in this sample, which was much smaller in size than for the other comparison performed for all LTNPs versus all other HIV-1+ individuals, shown in Table 2. Analysis of Kaplan-Meier life table curves for seroconvertors from the MACS cohort indicated that CCR5-2 heterozygotes had a small but statistically significant reduction in risk of develop- 
ing AIDS compared with the homozygous wildtype individuals. Dean et al. also reported a small effect of the CCR5-2 heterozygous genotype in delaying progression to AIDS for HIV-l+ gay men relative to homozygous wild-type CCR5 controls (12). Interestingly, they did not observe a significant difference in genotypic frequencies for cohorts of hemophiliacs segregated by rate of progression, which suggests that other factors can modulate the contribution of the CCR5-2 heterozygous genotype to the progression phenotype.

To understand these epidemiologic findings mechanistically, it will be important to correlate the frequency of CCR5-expressing cells and the level of CCR5 expression per cell both with viral replication in vitro and the rate of progression in infected patients, and to test whether these parameters differ in magnitude in CCR5-2 heterozygous versus wild-type homozygous individuals.

Taken together, the data suggest that the effect of gene dosage for wild-type CCR5 differs for initial infection versus clinical progression. Perhaps this is a consequence of differential expression of CCR5 in target cells in the mucosal barriers, where initial infection occurs, relative to lymphoid tissue that may be more important for amplification and spread of the virus. Detailed studies of CCR5 gene regulation and in vitro studies of viral replication in permissive cells from individuals homozygous for wild-type CCR5 and heterozygous for CCR5-2 are needed to clarify this finding.

Thus, the hypothesis that CCR5 is important for HIV-1 susceptibility and disease progression, which had as its origin the identification of HIV-1 coreceptor activity for CCR5 in vitro, has withstood a critical genetic test in populations of individuals having extremely polar clinical outcomes. The mutation in CCR5-2 causes disruption of the HIV-1 coreceptor activity and appears to confer high resistance to HIV-1, but only in homozygous individuals. This implies that other HIV-1 coreceptors are not sufficient for HIV-1 infection; whether they are necessary for HIV-1 infection or whether they affect the rate of disease progression requires further study. $\mathrm{Al}-$ though the relative rarity or absence of CCR5-2 homozygotes in the populations tested suggests that this mutation plays, at most, a minor role in checking the spread of the HIV-1 epidemic on a population level, the results also imply conversely that wild-type CCR5 may be an excellent target for the development of anti-HIV-1 drugs with broad clinical utility.

\section{ACKNOWLEDGMENTS}

We dedicate this study to Michael Chopek, M.D., our friend and colleage, who died suddenly on October 31, 1996. We thank M. Connor for patient samples; H.C. Lane, L. Schrager, J. Metcalf, S. Vogel, M. Polis, S. McCoy, C. Kleeberger, J. Phair, S. Wu, L. Jacobson, A. Munoz, and M. Majchrowicz for help in obtaining samples and for retrieval of coded clinical information; $R$. Plishka for DNA sequencing; M. Martin, J. Phair, J. Gallin, and H. Malech for helpful advice; D. Alling, C. Kleeberger, L. Jacobson, and A. Munoz for statistical advice; and the patients and clinical research staff of the MACS centers and the AIDS Clinic, NIAID, for making the DNA samples and clinical data analyzed in this study available. Samples and data provided by the Multicenter AIDS Cohort Study (MACS) were contributed by centers (principal investigators) at Johns Hopkins School of Public Health (A. Saah, A. Munoz, J. Margolick); Harold Brown Health Center and Northwestern University Medical School (J. Phair); University of California, Los Angeles ( $R$. Detels, J.V. Giorgi); and University of Pittsburgh (C. Rinaldo). The MACS is funded by the National Institute of Allergy and Infectious Diseases, with additional supplemental funding from the National Cancer Institute and the Agency for Health Care Policy and Research: UO1-AI-35042, 5-MO1-RR-00722 (GCRC), UO1AI-35043, UOl-AI-37984, UOl-AI-35039, UOlAI-37613, UOl-AI-35041.

\section{REFERENCES}

1. Murphy PM. (1996) Chemokine receptors: Structure, function and role in microbial pathogenesis. Cytokine Growth Fact. Rev. 7: 147-164.

2. Dragic T, Litwin V, Allaway GP, et al. (1996) HIV-1 entry into $\mathrm{CD}^{+}$cells is mediated by the chemokine receptor CC CKR-5. Nature 381: 667-673.

3. Alkhatib G, Combadiere C, Broder CC, et al. (1996) CC CKR5: A RANTES, MIP- $1 \alpha$, MIP- $1 \beta$ receptor as a fusion cofactor for macrophage-tropic HIV-1. Science 272: 19551958.

4. Choe H, Farzan M, Sun Y, et al. (1996) The 
$\beta$-chemokine receptors CCR3 and CCR5 facilitate infection by primary HIV isolates. Cell 85: 1135-1148.

5. Doranz BJ, Rucker J, Yi Y, et al. (1996) A dual tropic primary HIV-1 isolate that uses fusin and the $\beta$-chemokine receptors CKR-5, CKR-3, and CKR-2b as fusion co-factors. Cell 85: 1149-1158.

6. Deng H, Liu R, Ellmeier W, et al. (1996) Identification of a major co-receptor for primary isolates of HIV-1. Nature 381: 661-666.

7. Feng Y, Broder CC, Kennedy PE, Berger EA. (1996) HIV-1 entry co-factor: Functional cDNA cloning of a seven-transmembrane Gprotein coupled receptor. Science 272: 872877.

8. Bleul CC, Farzan M, Choe H, et al. (1996) The lymphocyte chemoattractant SDF-1 is a ligand for LESTR/fusin and blocks HIV-1 entry. Nature 382: 829-833.

9. Oberlin E, Amara A, Bachelerie F, et al. (1996) The CXC chemokine SDF-1 is the ligand for LESTR/fusin and prevents infection by T-cell-line-adapted HIV-1. Nature 382: 833-835.

10. Liu R, Paxton WA, Choe S, et al. (1996) Homozygous defect in HIV-1 coreceptor accounts for resistance of some multiply-exposed individuals to HIV-1 infection. Cell 86: 367-377.

11. Samson M, Libert F, Doranz BJ, et al. (1996) Resistance to HIV-1 infection in Caucasian individuals bearing mutant alleles of the CCR-5 chemokine receptor gene. Nature 382: 722-725.

12. Dean $M$, Carrington $M$, Winkler $C$, et al. (1996) Genetic restriction of HIV-l infection and progression to AIDS by a deletion allele of the CKR5 structural gene. Science 273: 1856-1861.

13. Connor RI, Ho DD. (1994) Human immunodeficiency virus type 1 variants with increased replicative capacity develop during the asymptomatic stage before disease progression. J. Virol. 68: 4400-4408.

14. Schuitemaker $\mathrm{H}$, Koot $M$, Kootstra NA, et al. (1992) Biological phenotype of human immunodeficiency virus type 1 clones at different stages of infection: Progression of disease is associated with a shift from a monocytotropic to T-cell tropic virus populations. J. Virol. 66: 1354-1360.

15. Loetscher $M$, Geiser $T$, O'Reilly $T$, Zwahlen R, Baggiolini M, Moser B. (1994) Cloning of a human seven-transmembrane domain re- ceptor, LESTR, that is highly expressed in leukocytes. J. Biol. Chem. 269: 232-237.

16. Combadiere C, Ahuja SK, Murphy PM. (1995) Cloning and functional expression of a human eosinophil CC chemokine receptor. J. Biol. Chem. 270: 16491-16494 [Erratum $J$ Biol Chem 270: 30235, 1995].

17. Kitaura M, Nakajima T, Imai T, et al. (1996) Molecular cloning of human eotaxin, an eosinophil-selective CC chemokine, and identification of a specific eosinophil eotaxin receptor, CC Chemokine Receptor 3. J. Biol. Chem. 271: 7725-7730.

18. Daugherty BL, Siciliano SJ, DeMartino JA, Malkowitz L, Sirotina A, Springer MS. (1996) Cloning, expression, and characterization of the human eosinophil eotaxin receptor. J. Exp. Med. 183: 2349-2354.

19. Ponath PD, Qin S, Post TW, et al. (1996) Molecular cloning and characterization of a human eotaxin receptor expressed selectively on eosinophils. J. Exp. Med. 183: 24372448.

20. Combadiere C, Ahuja SK, Tiffany HL, Murphy PM. (1996) Cloning and functional expression of CC CKR5, a human monocyte CC chemokine receptor selective for MIP-1 (alpha), MIP-1 (beta), and RANTES. J. Leukocyte Biol. 60: 147-152.

21. Raport CJ, Gosling J, Schweickart VL, Gray PW, Charo IF. (1996) Molecular cloning and functional characterization of a novel human CC chemokine receptor (CCR5) for RANTES, MIP-lbeta, and MIP-lalpha. J. Biol. Chem. 271: 17161-17166.

22. Paxton WA, Martin S, Tse D, et al. (1996) Relative resistance to HIV-1 infection of CD4 lymphocytes from persons who remain uninfected despite multiple high risk sexual exposures. Nature Med. 2: 412-417.

23. Munoz A, Kirby AJ, He YD, et al. (1995) Long-term survivors with HIV-1 infection: Incubation period and longitudinal patterns of $\mathrm{CD}^{+}$lymphocytes. Acquir. Immune Defic. Syndr. Hum. Retrovirol. 8: 496-505.

24. Detels R, Liu Z, Hennessey K, et al. (1994) Resistance to HIV-l infection. Multicenter AIDS Cohort Study. J. Acquir. Immune Defic. Syndr. 7: 1263-1269.

25. Imagawa DT, Lee $\mathrm{MH}$, Wolinsky SM, et al. (1989) Human immunodeficiency virus type 1 infection in homosexual men who remain seronegative for prolonged periods. $N$. Engl. J. Med. 320: 1458-1462.

26. Zimmerman PA, Phadke PM, Lee A, et al. 
(1995) Migration of a novel DQA * allele $\left(\mathrm{DQAl}{ }^{*} 0502\right)$ from African origin to North and South America. Hum. Immunol. 42: 233240.

27. Zimmerman PA, Steiner LL, Titanji VPK, et al. (1996) Three new DPBI alleles identified in a Bantu-speaking population from central Cameroon. Tissue Antigens 47: 293-299.

28. Zimmerman PA, Carrington MN, Nutman TB. (1993) Exploiting structural differences among heteroduplex molecules to simplify genotyping the DQAl and DQB1 alleles in human lymphocyte typing. Nucl. Acids Res. 21: 4541-4547.

29. Nutman TB, Zimmerman PA, Kubofcik J, Kostyu D. (1994) A universally applicable diagnostic approach to filarial and other infections. Parasitol. Today 10: 239-243.

30. Ng J, Hurley CK, Baxter-Lowe LA, et al. (1993) Large-scale oligonucleotide typing for HLA-DRB 1/3/4 and HLA-DQB1 is highly accurate, specific, and reliable. Tissue Antigens 42: 473-479.

31. Nussbaum O, Broder CC, Berger EA. (1994) Fusogenic mechanisms of enveloped-virus glycoproteins analyzed by a novel recombinant vaccinia virus-based assay quantitating cell fusion-dependent reporter gene activation. J. Virol. 68: 541 1-5422.

32. Samson M, Labbe O, Mollereau C, Vassart G, Parmentier M. (1996) Molecular cloning and functional expression of a new human CC chemokine receptor gene. Biochemistry 35: 3362-3367.

33. Ahuja SK, Lee JC, Murphy PM. (1995) CXC chemokines bind to unique sets of selectivity determinants that can function independently and are broadly distributed on multiple domains of human interleukin-8 receptor B. Determinants of high affinity binding and receptor activation are distinct. J. Biol. Chem. 271: 225-232.

34. Cocchi F, DeVico AL, Garzino-Demo A, Arya SK, Gallo RC, Lusso P. (1995) Identification of RANTES, MIP- $1 \alpha$, and MIP- $1 \beta$ as the major HIV-suppressive factors produced by $\mathrm{CD}^{+}{ }^{+}$cells. Science 270: $1811-1815$.

35. Miller LH, Mason SJ, Dvorak JA, McGinniss MH, Rothman IK. (1975) Erythrocyte receptors for (Plasmodium knowlesi) malaria: Duffy blood group determinants. Science 189: 561563.

36. Horuk R, Chitnis CE, Darbonne WC, et al. (1993) The erythrocyte chemokine receptor is a receptor for the malarial parasite Plasmodium vivax. Science 261: 1182-1184.

37. Horuk R, Wang ZX, Peiper SC, Hesselgesser J. (1994) Identification and characterization of a promiscuous chemokine-binding protein in a human erythroleukemic cell line. J. Biol. Chem. 269: 17730-17733.

Communicated by A. Fauci. Accepted on November 8, 1996. 